\title{
Untersuchungen zum ionisierten Calcium im Humanserum Normalwerte und Belastungsprüfungen (Calcium, Parathormon, Calcitonin)
}

\author{
Von P. O. Schiville, F. Gniffke, A. Sigel und H. Bünte \\ Aus dem Laboratorium der Chirurgischen Klinik und Abteilung Urologie der Universität Erlangen-Nürnberg
}

(Eingegangen am 18. April/23. August 1972)

\begin{abstract}
Ionisiertes Calcium im menschlichen Serum wird mittels ionensenșitiver Elektrode bestimmt: Außerdem werden Gesamtcalcium, sein ultrafiltrierbarer Anteil und Gesamteiweiß mit herkömmlichen Methoden erfaßt. Der Mittelwert des ionisierten Calciums bei 21 Gesunden aus 2 verschiedenen Altersgruppen liegt bei $1,13 \pm 0,073 \mathrm{mmol} / \mathrm{l}$. Es finden sich keine signifikanten Unterschiede im relativen Anteil am Gesamtcalcium zwischen Gesunden, Patienten mit sog. idiopathischer Hypercalciurie und solchen mit primärem Hyperparathyreoidismus. Nach i. v. Infusion von $15 \mathrm{mg} / \mathrm{kg}$ Calciumionen wird der geringste Anstieg des ionisierten Anteils bei Hyperparathyreoidismus beobachtet, ein etwas stärkeret bei Hypercalciurie. Nach einmaliger i. v. Injektion von 0,8 E/kg Parathormon fällt Gesamtcalcium bei Gesunden initial signifikant ab, während umgekehrt das ionisierte Calcium gering ansteigt (n. s.). Nach einmaliger i. v. Injektion von 0,2 MIRC-U/kg Calcitonin kommt es bei Gesunden um die 45. Minute post inj. zu einem signifikanten Anstieg des ionisierten Calciums (Absolutwerte) und bei Hyperparathyreoidismus ist dieses Verhalten noch deutlicher ausgeprägt. Aufgrund der Ergebnisse wird vermutet, daß Calcitonin zelluläres Parathormon freizusetzen vermag.
\end{abstract}

\section{Ionized calcium in buman serum. Normal values and loading tests (calcium, paratbyroid bormone, calcitonin)}

Ionized calcium in human serum was determined by ion sensitive electrode. Total and ultrafiltrablecalcium and total protein were also analyzed by conventional methods. The aim of this work was to study ionized calcium under various experimental conditions (i. $v$. infusion of calcium gluconicum; $15 \mathrm{mg} \mathrm{Ca}{ }^{++} / \mathrm{kg}$; single i. v. injection of $0.8 \mathrm{U} / \mathrm{kg}$ parathyroid hormone; single i. v. injection of $0.2 \mathrm{MRC}-\mathrm{U} / \mathrm{kg}$ calcitonin). The mean value in healthy volunteers ( 2 age groups, $n=21$ ) was $1.13 \pm 0.073 \mathrm{mmol} / 1$. Patients suffering from idiopathic hypercalciuria and primary hyperparathyroidism demonstrate a smaller increase of ionized calcium after calcium infusion than healthy volunteers. After administration of parathyroid hormone in volunteets, total calcium initially decreases significantly, ionized calcium (absolute values) remaining invariable. Calcitonin in the same group, however, provoked a significant increase in absolute values of ionized calcium at $45 \mathrm{~min}$ post inj. and a more pronounced one at $90 \mathrm{~min}$ in the hyperparathyroid group. The findings would be suggestive for a direct liberation of parathyroid hormone induced by calcitonin.

Wegen seiner großen biologischen Bedeutung und der Variabilität seiner Fraktionen im Serum ist Calcium ein interessantes Kation. In der Vergangenheit beschäftigten sich zahlreiche Untersuchungen mit der Technik der Auftrennung in proteingebundenen und ultrafiltrierbaren Anteil, während nur wenige die Erfassung des allein biologisch aktiven ionisierten Calciums $\left(\mathrm{Ca}^{++}\right)$ zum Gegenstand hatten $(1,2,3)$. Die Froschherzmethode (4) stellt ein äußerst empfindliches Meßprinzip dar, ist jedoch technisch schwer durchführbar und für klinische Bedingungen ungeeignet. Das zunehmende Auftreten von Mineralstoffwechselstörungen mit charakteristischen Merkmalen (primärer und sekundärer Hyperparathyreoidismus, Osteomalazie, rezidivierende Nephrolithiasis usw.) verlangt geeignete Methoden zur Messung des ionisierten Anteils, der über einen negativen Rückkoppelungsmechanismus durch Parathormon und Calcitonin eng reguliert wird (5).

In jüngerer Zeit stehen Bemühungen im Vordergrund, diesen Anteil über sensitive Elektroden elektrometrisch zu erfassen (6). Im Handel befindliche Systeme ${ }^{1}$ ) erlauben unter Einhaltung bestimmter Kautelen eine zuverlässige Messung und erscheinen damit auch für den

1) z. B. Fa. Orion, Richmond (USA); deutscher Vertrieb: Colora $\mathrm{GmbH}$, Lorch. klinischen Gebrauch geeignet. In dieser Untersuchung haben wir folgende Fragen geprüft:

1. die Genauigkeit der Methode (Reproduzierbarkeit, Wiederfindungsrate);

2. in welchem Bereich liegen die Normalwerte Gesunder;

3. Untersuchung eines klinischen Patientengutes (aktuelle Werte, Verhalten unter einer Calcium-Infüsion, nach einmaliger Injektion einer kleinen Dosis Parathormon bzw. Calcitonin).

\section{Material und Methoden}

Zur Untersuchung kamen gesunde Versuchspersonen aus 2 verschiedenen Altexsgruppen, sowie Patienten:

$\mathrm{P}_{1}$ : 9 gesunde Probanden, Alter $20-30$ Jahre;

$\mathrm{P}_{2}: 12$ gesunde Probanden, Alter 30-60 Jahre;

HC: 20 Patienten mit sog. idiopathischer Hypercalciurie (operationsbedürftige Oxalatkonkremente);

O: 10 Patienten mit dem Symptom des rezidivierenden Spontanabgangs kleiner Oxalatkonkremente;

I: Patienten mit infektbedingter Nephrolithiasis (Tripelphosphat); HPT: 5 Patienten mit primärem Hyperparathyreoidismus, operativbioptisch gesichert.

Die Anzahl Versuchspersonen in den Gruppen bezieht sich auf die Erstellung von Normalwiverten (s. Abb. 3).

Gemessen wurde im Serum unter Einhaltung definierter Bedingungen: $12 \mathrm{~h}$ nüchtern, keine körperliche Belastung, Adaptation 
der Patienten an calciumarme Kost während 1 Woche, Blutentnahme aus ungestauten Venen, Uberschichtung der Proben mit ol. paraffini bereits während der Aspiration.

Die Calciumbelastung erfolgte nach dem von KrLe (7) erstmals beschriebenen Vorgehen zur Prüfung der Supprimierbarkeit der Nebenschilddrüsen (6 gesunde Probanden, zusammen $19 \mathrm{~Pa}$ tienten aus den Gruppen HC, O, I und HPT): i. v. Infusion von $15 \mathrm{mg} / \mathrm{kg}$ Calciumionen ${ }^{2}$ ) in $500 \mathrm{ml} \mathrm{NaCl}$-Lösung ( $150 \mathrm{mmol} / \mathrm{l}$ ) während $3 \mathrm{~h}\left(21^{00}-24^{00}\right)$. Parathormonbelastung $\left.{ }^{3}\right)$ : nach $\mathrm{Ab}-$ nahme von wenigstens 2 Kontrollwerten einmalige i. v. Injektion von $0,8 \mathrm{E} / \mathrm{kg}$ (10 gesunde Probanden). Calcitoninbelastung $\left.{ }^{4}\right)$ : wie unter Parathormon, einmalige i. v. Injektion von 0,2 MRC U/kg (10 gesunde Probanden und 6 Fälle von primärem Hyperparathyreoidismus).

Bestinmungen: Ionisiertes Calcium mittels Durchflußelektrode (Orion), Gesamtcalcium und ultrafiltrierbarer Anteil komplexometrisch (Indikator Calcein), sowie Ultrafiltration nach früher beschriebenen Verfahren $(8,9)$, Gesamteiweiß im Serum über Standardmethode Autoanalyzer (Technicon).

Statistiscbe Auswertung: Prüfung auf Normalverteilung (KolmoGOROFF-SMIRNOW), auf Signifikanz (t-Test nach Studenr: Mittelwerte \pm 1 fache Standardabweichung), lineare Regression.

\section{Ergebnisse}

\section{Genavigkeit der Metbode}

Bei Bestimmungen in der Serie (gleiche Serumprobe an einem Tag, $\mathbf{n}=10$ ) schwanken die Werte geringfügig: $1,13 \pm 0,025 \mathrm{mmol} / \mathrm{l}, \mathrm{VK} 2,2 \%$.

Wegen der mit zunehmendem Calciumgehalt steigenden Calciumbindung an Serumproteine eignet sich weder Poolserum (normales Serumcalcium) noch Hypoparathyreoidismusserum für die Prüfung auf Wiederfindung, dagegen besteht eine fast lineare Beziehung zwischen Vorlage und Wiederfindung im Ultrafiltrat, worauf bereits Moore (6) hingewiesen hatte (Abb. 1). Die im Serum und zugehörigen Ultrafiltrat gleichzeitig gemessenen Werte differieren ${ }_{\alpha}[$ jedoch erheblich in Abhängigkeit vom Eiweiß/Albumingehalt: in Abbil-

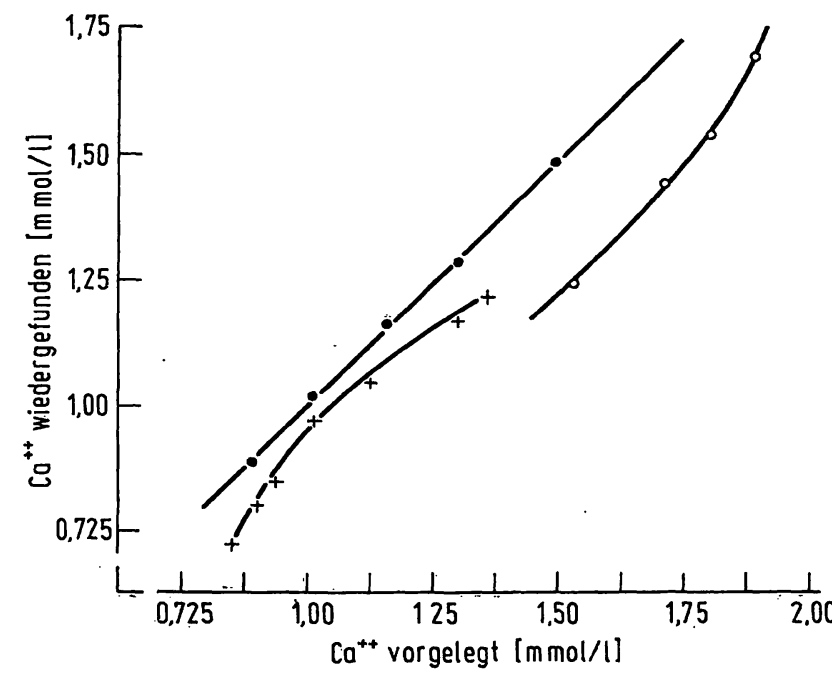

Abb. 1

Bestimmung von ionisiertem Calcium $\left(\mathrm{Ca}^{++}\right)$im menschlichen Serum mittels sensitiver Elektrode (O-O: Poolserum klinisch Gesunder, $+-+:$ Hypoparathyreoidismusserum, - Ultrafiltrat). WiederIonenstärke $\left(2,0 \mathrm{mmol} / 1 \quad \mathrm{CaCl}_{2}, 150 \mathrm{mmol} / 1 \mathrm{NaCl}\right)$ zum Ausgangsmaterial

2) Calcium gluconicum (Sandoz AG, Nürnberg).

3) Parathormon Lilly (Lilly GmbH, Bad Homburg).

4) Calcitonin vom Schwein (Organon $\mathrm{GmbH}$, München).

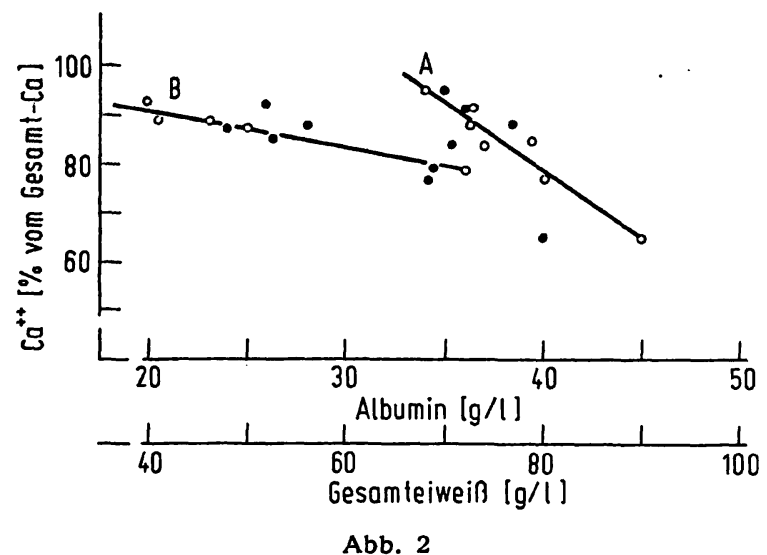

Ionisiertes Calcium im Ultrafiltrat ( $\mathrm{pH} \sim 7,40$ ) in \% des zugehörigen Serumwertes (Ordinate) in Abhängigkeit von der Konzentration an Gesamteiweiß bzw. Albumin im Serum (Abszisse). O-O: Albumin,

A: Nativserum ( $r=-0,78$, $p>0,05$, bezogen auf Albumin),

B: Verdünnte Serumproben (Calciumchloridlösung: 2,0 mmol/l $\mathrm{CaCl}_{2}, 150 \mathrm{mmol} /(\mathrm{NaCl}) . \mathrm{r}=-0,91, \mathrm{p}<0,01$, bezogen auf Albumin

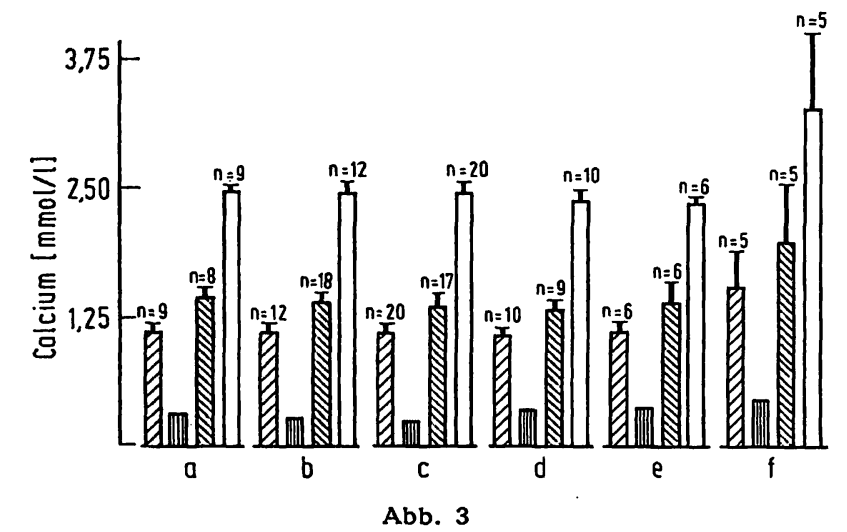

Calciumfraktionen im Humanserum. WIISY: ionisiertes Calcium, ultrafiltrierbares Calcium, IIIII): an Komplexe gebundenes Calcium $=\mathrm{Ca}_{\mathrm{d}}-\mathrm{Ca}^{++}$, $\square:$ Gesamtcalcium

a: gesunde Probanden (Alter 20-30 Jahre)

b: gesunde Probanden (Alter 30-60 Jahre)

c: Patienten mit Hypercalciurie

d: Patienten mit Spontanabgang von Oxalatkonkrementen

: infektbedingte Nephrolithiasis

$\mathrm{f}$ : primärer Hyperparathyreoidismus

dung 2 ist das im Ultrafiltrat gemessene ionisierte Calcium in Prozent des Serumwertes gegen die jeweilige Konzentration an Gesamteiweiß bzw. Albumin aufgetragen.

\section{Die aktuellen Werte der geprïften Gruppen}

In Abbildung 3 sind die Calciumfraktionen gesunder Probanden und Patienten wiedergegeben. Die Mittelwerte des ionisierten Calciums aller Gruppen, ausgenommen Hyperparathyreoidismus, unterscheiden sich nur geringfügig:

$P_{1}$ (20-30 Jahre) $1,13 \pm 0,075$;

$\mathrm{P}_{2}$ (30-60 Jahre) 1,13 $\pm 0,075$;

HC (Hypercalciurie) $1,13 \pm 0,075$;

O (kleine Oxalatkonkremente) $1,10 \pm 0,075$;

I (Infektlithiasis) 1,15 $\pm 0,05$, jeweils $\mathrm{mmol} / \mathrm{l}$.

Bei primärem Hyperparathyreoidismus liegen zwar die absoluten Werte signifikant höher (1,58 $\pm 0,35 \mathrm{mmol} / \mathrm{l})$, jedoch sind die prozentualen Anteile am Gesamtcalcium in allen Gruppen nahezu gleich groß (Tab. 1). 
Tab. 1

Anteile (in \%) von ionisiertem (1) und ultrafiltrierbarem (2) Calcium am Gesamtcalcium und Anteil des ionisierten am ultrafiltrierbaren (3). Mittelwerte und Standardabweichung. ( ): Anzahl der Versuchspersonen, $\mathrm{Ca}^{++}$: ionisiertes Calcium, $\mathrm{Ca}_{\mathrm{g}}$ : Gesamtcalcium, Ca $\mathrm{a}_{\mathrm{d}}$ : ultrafiltrierbares Calcium

\begin{tabular}{|c|c|c|c|}
\hline Gruppe & $\begin{array}{c}(1) \\
\frac{\mathrm{Ca}^{++}}{\mathrm{Ca}_{\mathrm{g}}} \cdot 100\end{array}$ & $\begin{array}{c}(2) \\
\frac{C a_{d}}{C a_{g}} \cdot 100\end{array}$ & $\begin{array}{c}(3) \\
\frac{\mathrm{Ca}^{++}}{C a_{d}} \cdot 100\end{array}$ \\
\hline $\begin{array}{l}\text { Gesunde Probanden (Alter } 20-30 \mathrm{~J} \text { ) } \\
\text { Gesunde Probanden (Alter } 30-60 \mathrm{~J} . \text { ) } \\
\text { Hypercalciurie } \\
\text { Kleine Oxalatkonkremente (Spontanabgang) } \\
\text { Infektbedingte Nephrolithiasis } \\
\text { Primärer Hyperparathyreoidismus }\end{array}$ & $\begin{array}{r}45,6 \pm 3,10(9) \\
44,9 \pm 3,69(13) \\
45,6 \pm 2,94(20) \\
46,10 \pm 2,90(10) \\
48,30 \pm 2,70(3) \\
48,0 \pm 3,32(5)\end{array}$ & $\begin{array}{l}59,38 \pm 3,46(8) \\
- \\
55,90 \pm 5,40(17) \\
56,80 \pm 2,90(9) \\
60,60 \pm 6,90(3) \\
62,0 \pm 3,30(5)\end{array}$ & $\begin{array}{c}77,0 \pm 8,50(8) \\
- \\
82,40 \pm 8,24(17) \\
83,60 \pm 6,50(9) \\
80,66 \pm 7,79(3) \\
79,0 \pm 6,96(5)\end{array}$ \\
\hline
\end{tabular}

\section{Verbalten unter der Calciuminfusion}

In Abbildung 4 ist das ionisierte Calcium in Prozent des jewreiligen Gesamtcalciums im Tagesablauf bzw. unter dem Einfluß der Infusion dargestellt. Signifikante Unterschiede ergeben sich für die einzelnen. Gruppen nicht, doch findet sich ein deutlicher Unterschied zwischen Gesunden $\left(P_{1}\right)$ und Patienten mit infektbedingter Nephrolithiasis (I) einerseits und den restlichen Gruppen (HC, O, HPT) andererseits, deren gemeinsames Merkmal eine mehr/minder ausgeprägte Hypercalciurie und Neigung zur Oxalatkonkrementbildung in den Nieren ist.

\section{Paratbormonbelastung}

Das Verhalten nach einmaliger i. v. Injektion einer kleinen Dosis (Gruppe $\mathrm{P}_{2}: 30-60$ Jahre) ist in $\mathrm{Ab}-$ bildung 5 dargestellt. Neben einem uncharakteristischen geringen Anstieg des ionisierten Anteils imponiert der scharfe initiale Abfall des Gesamtcalciums. Dieser ist zwar unter Verwendung weit größerer Hormondosen im Tierexperiment $(10,11)$ und am Menschen (12) schon früher beobachtet worden, doch konnten wir den gleichen Effekt unter höherer Dosierung des handelsüblichen Extrakts (Lilly) nicht reproduzieren (unveröffentlicht).

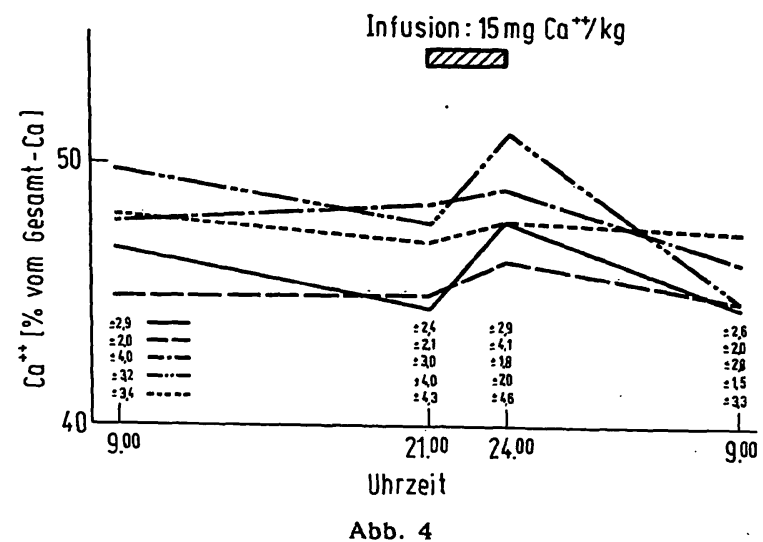

Das Verhalten der verschiedenen Gruppen unter der Calciuminfusion nach KYLE (7). Mittelwerte des ionisierten Calciums $\left(\mathrm{Ca}^{++}\right)$in $\%$ des
Gesamtcalciums der jeweiligen Uhrzeit. Standardabweichung in Zahlen

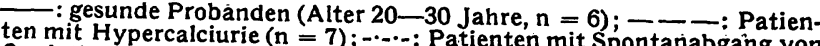
ten mit Hypercalciurie $(n=7) ; \cdots \cdot \cdots$ : Patienten mit Spontanabgang von $(n=3) ; \cdots \cdots$ primärer Hyperparathyreoidismus $(n=5)$

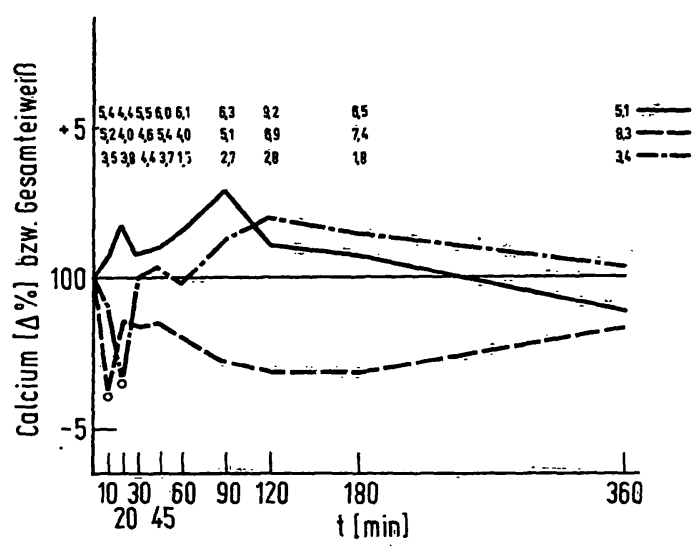

Abb. 5

Das Verhalten von ionisiertem Calcium (-), Gesamtcalcium $(-\cdot)$ und Gesamteiweiß (--) im Serum gesunder Probanden (Alter 30 bis $60 \mathrm{Jahre}, \mathrm{n}=10$ ) nach einmaliger $\mathrm{i}$. v.-Injektion von $0,8 \mathrm{E} / \mathrm{kg}$ Parathormon (Lilly). Abweichungen in Prozent vom Ausgangswert (Mittelhormon (Lilly). Abweichungen in Prozent vom Ausgangswert (Mittel-
wert aus 2 4 Kontrollwerten $=100$ ). Standardabweichung in Ausgangswerte: ionisien. $O: \mathrm{p}<0,025$ ngswerte: ionisiertes Calcium $-1,075 \pm 0,075 \mathrm{mmol} / ; \mathrm{Ge}-$
samtcalcium $-2,475 \pm 0,125 \mathrm{mmol} / 1 ;$ Gesamteiweiß

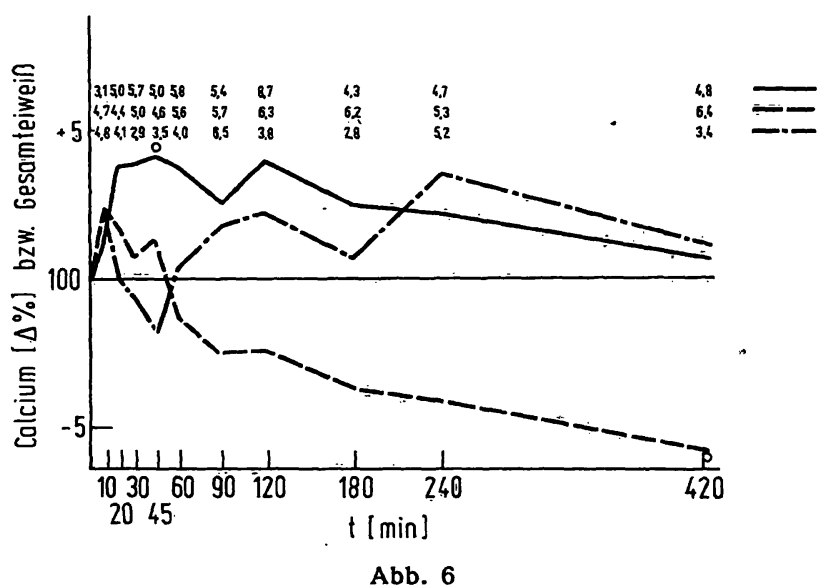

Das Verhalten von ionislertem Calcium $(-))$ Gesamtcalcium $\left(--\frac{-}{30}\right)$ und Gesamteiweiß (--) im Serum gesunder Probanden (Alter 30-60 Jahre, $n=10$ ) nach einmaliger $i$. v.-Injektion von $0,2 \mathrm{MCR}-\mathrm{U} / \mathbf{k g}$ Calcitonin (vom Schwein). Abweichungen in Prozent vom Ausgangswert (Mittelwert aus 2-4 Kontrollwerten = 100). Standardabweichung in Zahlen. O: $p$ < 0,01 Ausgangswerte: ionisiertes Cälcium Gesamteiwei $3-83,0 \pm 11,3 \mathrm{~g} / 1$

\section{Calcitoninbelastung}

a) Abbildung 6 - Vorgehen wie für Parathormon beschrieben (Gruppe $P_{2}$ ). Im Vergleich zum Verlauf unter Parathormon kommt es initial zu einem nahezu gegensätzlichen Verhalten von Gesamtcalcium und -eiweiß (Anstieg). Umgekehrt strebt der ionisierte 


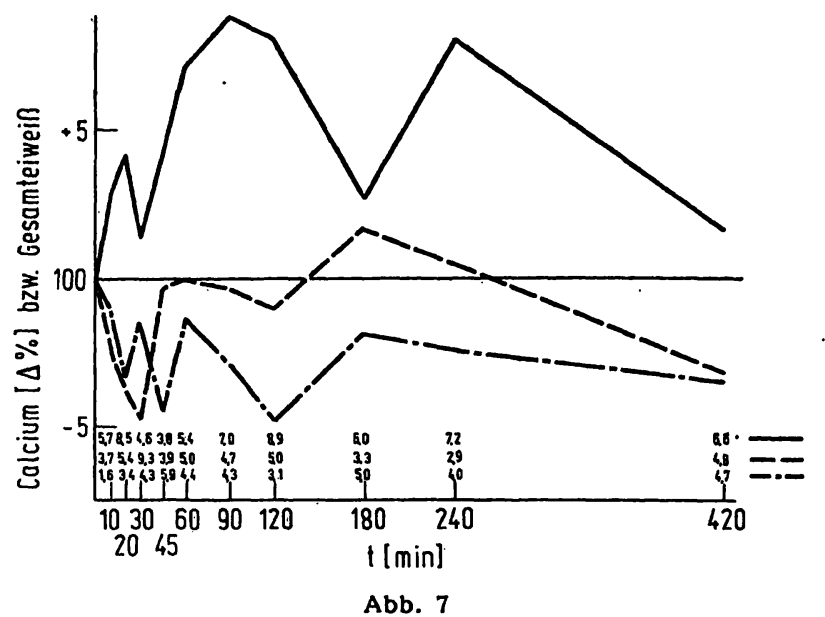

Das Verhalten von ionisiertem Calcium (-), Gesamtcalcium (- und Gesamteiweiß (--) im Serum bei primärem Hyperparathyreoidismus $(n=6)$ nach einmaliger $i$. v.-Injektion von $0,2 \mathrm{MRC}$-U/kg Calcitonin (vom Schwein). Abweichungen in Prozent vom Ausgangswert (Mittelwert aus 3 Kontrollwerten $=100)$. Standardabweichung in Zahlen. $O: p<0,01,0: p<0,05$ Ausgangswerte: ionisiertes Calcium $-1,275 \pm 0,175 \mathrm{mmol} / 1 ;$
samtcalcium $-2,95 \pm 0,625 \mathrm{mmol} / 1 ;$ Gesamteiweiß $-75,0 \pm 5,0 \mathrm{~g} / \mathrm{l}$

Anteil einem signifikant höheren Maximalwert um die 45. Minute zu.

b) Abbildung 7 - primärer Hyperparathyreoidismus: Gesamtcalcium und -eiweiß fallen frühzeitig (30. bis 45. Minute) deutlich ab (nicht signifikant), während der ionisierte Anteil nachhaltig ansteigt und 2 Maximalwerte erreicht (90. und 240. Minute).

\section{Diskussion}

Über die elektrometrische Bestimmung des ionisierten Calciums im Serum mittels sensitiver Elektrode liegen bisher mehrere Publikationen vor. Unsere aktuellen Werte stimmen gut überein mit jenen von MOORE (6) und RAman (13), liegen aber etwa $10 \%$ niedriger als die zuletzt von Fuchs (14) mitgeteilten. Von Pedersen (15) wurden spektrophotometrisch ebenfalls $1,14 \mathrm{mmol} / \mathrm{l}$ bestimmt, und für den Gleichgewichtszustand zwischen Parathormon und Calcitonin (bei Schwein und Schaf) nannte Copp (16) zuletzt $1,18 \mathrm{mmol} / \mathrm{l}$.

Die künftige Erfahrung wird zeigen, ob die ursprünglich vorgeschlagene Verwendung von paraffinüberschịchteten Proben einen $\mathrm{CO}_{2}$-Verlust aus dem Plasma mit folgendem $\mathrm{pH}=$ Anstieg nach sich zieht.

Nieedrigere Meßwerte ließen sich hierdurch erklären. Gegen die alleinige Brauchbarkeit von unter Vakuum abgenommenen Proben würde sprechen, daß bereits mit einer früheren Meßanordnung (Stativelektrode, aerobes Vorgehen) Werte zwischen 1,23-1,28 mmol/l erhoben wurden (17). Da Magnesium und Kalium im Serum nur einen geringen Einfluß auf die Ionenstärke des Probenmaterials ausüben, erscheint die auschließliche Verwendung entsprechend zubereiteter Eichlösungen zur Zeit nicht gerechtfertigt. Als Nachteil muß bezeichnet werden, $\mathrm{da}$ eine ausreichende Qualitätskontrolle im Serum nicht möglich ist (Wiederfindungsrate). Andererseits beweist die Wiederfindungstate in eiweißfreiem Material methodische Richtigkeit (Abb. 1). Vor allem technische Schwierigkeiten bei der Kon- servierung eines konstanten $\mathrm{pH}$ im Ultrafiltrat und der unterschiedliche Eiweißgehalt der Serumproben (Abb. 2) stehen der alleinigen Messung im Ultrafiltrat entgegen. Deshalb muß geprüft werden, ob unter Beachtung der individuellen Eiweißkonzentration eine Interpolation der Serumwerte aus jenen im Ultrafiltrat möglich bzw. statthaft ist.

\section{Calciuminfusion}

Der in der Gruppe Hyperparathyreoidismus deutlich geringe Anstieg des ionisierten Calciums am Ende der Infusion wird von uns auf die bei solchen Kranken gesteigerte skelettale Umbaurate (turn over) zurückgeführt. Trotz des unterschiedlichen Verhaltens der einzelnen Gruppen erlauben die Daten keine Differentialdiagnose, insbesondere besteht zwischen den Gruppen HC (Hypercalciurie) und HPT (Hyperparathyreoidismus) ein beträchtlicher Überlappungsbereich. Da aber beide Gruppen mit dem Symptom der Oxalatkonkrementbildung $(\mathrm{O}, \mathrm{HC})$ von Gesunden deutlich abweichen, sollte die Natur der sog. ,idiopathischen Hypercalciurie" erneut unter dem Gesichtspunkt der Beteiligung des Skeletts studiert werden, worüber erheblich unterschiedliche Auffassungen bestehen (18).

\section{Parathormonbelastung}

Sie wurde ebenso wie die Calcitoninbelastung durch die zuletzt von Parsons et al. (10) gemachte Beobachtung initiiert, wonach auch hochgereinigtes bovines Parathormon sehr früh einen zellulären Ca-Influx und konsekutiven Abfall des Gesamtcalciums im Serum vermittelt. Eine begleitende Calcitoninbeimengung als Ursache dieses Effekts scheidet damit aus. Untersuchungen am Menschen unter Verwendung kleiner Hormondosen, wie in dieser Studie, sind uns bisher nicht bekannt geworden. Daher waren 3 Fragen von Interesse :

1. Läßt sich ein frühzeitiger Calcium-Abfall auch unter diesen Umständen objektivieren. Falls dies zutrifft, so wäre wenig wahrscheinlich, daß es sich in Wirklichkeit um einen Calcitonineffekt des handelsüblichen Extrakts handelt.

2. Umgekehrt müßte eine kleine Dosis Calcitonin qualitativ gegenteilige Effekte hervorrufen.

3. Welche Änderungen erfährt das ionisierte Calcium, das alleine den negativen Rückkoppelungsmechanismus zwischen Nebenschilddrüsen- und C-Zellaktivität unterhält.

$\mathrm{Zu}$ 1.: Der auch von uns beobachtete, rasch einsetzende Abfall des Gesamtcalciums unter Parathormon muß auf Kosten der an Komplexe gebundenen ultrafiltrierbaren Calciumfraktion zustande kommen, da zeitgleich auch das Serumprotein abfällt und das ionisierte Calcium geringfügig ansteigt (n. s.). Erst ab der 30 . Minute kommt es zu einer nahezu spiegelbildlichen Dissoziation zwischen Gesamtcalcium und -eiweiß, die wir als 2. Phase der Hormonwirkung bezeichnen. Diese Konstellation ist in der Differentialdiagnose der Hypercalcämie klinisch von erheblichem Interesse. 


\section{Zu. 2.: Calcitoninbelastung}

In Analogie zur zitierten und experimentell belegten Theorie des Parathormon-vermittelten Ca-Influxes (10) sieht man unter Calcitonin zunächst einen Anstieg von Gesamtcalcium und -eiweiß (nicht signifikant), der als Ausdruck des zellulären Ca-Effluxes gewertet werden kann. Der signifikante Anstieg des ionisierten Anteils bei Gesunden (Abb. 6) steht jedoch im Widerspruch zu den meisten bisherigen Mitteilungen, wonach es unter Calcitonin regelmäßig zur Calciumerniedrigung kommt. $\mathrm{Da}$ meistens sehr hohe Hormonmengen verabreicht wurden, besteht der Verdacht, daß dieser geläufige Calciumabfall nach Calcitonin zumindest zum Teil auf pharmakologischen Effekten der jeweiligen Präparation beruht oder auf eine gesteigerte Empfindlichkeit der verwendeten Versuchstierspezies zurückgeht. Der von uns beobachtete Anstieg des ionisierten Calciums (Frage 3) kann Ausdruck eines veränderten transzellulären Transports sein und nachfolgender Erhöhung des Ionisationsgrades auf Kosten des komplex gebundenen Anteils. Außerdem könnte es über Triggermechanismen zur Liberation gespeicherten Parathormons kommen. Für letzteres würden 2 Umstände sprechen: einmal sieht man auch unter Calcitonin bei Gesunden $a b$ der 45. Minute einen nachhaltigen Anstieg des Gesamtcalciums (nicht signifikant) ähnlich jenem nach Parathormon (Abb. 5), zum anderen berichteten FISCHER et al. (19) über eine direkte Calci- toninbedingte Freisetzung von Parathormon im in vitro-System. Andere Ursachen für den Anstieg des ionisierten Anteils wie metabolische Einflüsse (Erniedrigung des Serum-pH) scheiden nach unseren Untersuchungen aus. Die Vermutung, daß Calcitonin Parathormon freizusetzen vermag, wird außerdem gestützt durch die bei HyperparathyreoidismusPatienten erhobenen Befunde (Abb. 7): Gesamtcalcium und -eiweiß fallen initial wie nach Parathormon-Injektion bei Gesunden (Abb. 5) deutlich ab, während der ionisierte Anteil noch ausgeprägter ansteigt als nach Calcitoningabe bei Gesunden (Abb. 6). Dieser unerwartete Verlauf ist vereinbar mit der erhöhten Konzentration an Parathormon bei Hyperparathyreoidismụs, könnte aber auch auf ein endogenes Calcitonindefizit (20) und gesteigerte Ansprechbarkeit auf kleine (Trigger)dosen des Hormons bei diesen Kranken hinweisen. Für den Verlauf des Gesamtcalciums dieser Patienten unter den Ausgangswerten auch nach der 45.-60. Minute haben wir zunächst keine Erklärung. Weitere Untersuchungen müssen zeigen, ob eine Calcitoninbelastung in der Differentialdiagnose der Hypercalcämie ein brauchbares Hilfsmittel wird.

\section{Danksagung}

Den Firmen Lilly GmbH, Bad Homburg, Organon GmbH, München und Colora GmbH, Lorch, sind wir für die Überlassung von Substanzen bzw. Geräten zu Dank verpflichtet.

\section{Literatur}

1. McLean, F. C. \& Hastings, A. (1935), J. Biol. Chem. 108, 285-290. - 2. LunrB, G. A. (1963), Clin. Chim. Acta 8, 33-38. 3. RaAflaub, J. (1951), Hoppe-Seyler's Z. Physiol. Chem. 288, 228-251. - 4. AICLEAN, F. C. \& Hastings, A. (1934), J. Biol. Chem. 107, 337-344. - 5. Copp, D. H. (1968), in Parathyroid Hormone and Thyrocalcitonin: Talmage, R. W. \& Bélanger, H. F., Eds. Excerpta Medica Foundation, New York. - 6. MOORE, E. (1970), J. Clin. Invest. 49, 318-334. - 7. KyLE, L. H., CANARY, J. J., Mintz, D. H. \& DE LeON, A. (1962), J. Clin. Endocr. 22, 52-64. - 8. Schwille, P. O. \& ERnstberger, W. (1972), Clin. Chim. Acta 38, 379-384. - 9. Pfordte, K. \& Ponsold, W. (1967), Zschr. ges. Exp. Med. 144, 31-38. - 10. Parsons, J. A. NeER, R. M. \& Potrs jr., J. T. (1971), Endocrinology 89, 735 bis 740. - 11. Copp, D. H., Cameron, E. C., Cheney, B. A., David- son, A. G. F. \& HeNZE, K. G. (1962), Endocrinology 70, 638 bis 649. - 12. HaAs, H. G., Affolter, H. \& Dubach, U. C. (1961), Acta Endocr. 48, 132-136. - 13. RAman, A. (1970), Biochem. Med. 3, 369-375. - 14. Fuchs, C. \& PAsCHeN, K. (1972), Deut. med. Wochenschr. 97, 23-24. - 15. PEDERSEN, K. O. (1970), Scand. J. clin. Lab. Invest. 25, 223-230. - 16. Copp, D. H. (1970), Ann. Rev. Phys. 32, 61-86. - 17. Arnold, D. H., Stansele, M. J. \& Malvin, H. H. (1968), Am. J. Clin. Path. 49, 627-634. - 18. HaAs, H. G. (1966), in Parathyreoidea und Knochenerkrankungen, S. 99, Thieme-Verlag, Stuttgart. -19. Fischer, J. A., Oldham, S. B., Sizemore, G. W. \& Arnaud, C. D. (1971), Horm. Metab. Res. 3, 223-224. - 20. Tashjian jr., A. H. \& Voelked, E. F. (1967), J. Clin. Endocr. Metab. 27, 1353-1357.
Dr. P. O. Schwille

Mineralstofflabor der Chirurg. Univ.-Klinik und Abteilung Urologie

852 Erlangen

Krankenhausstr. 12 


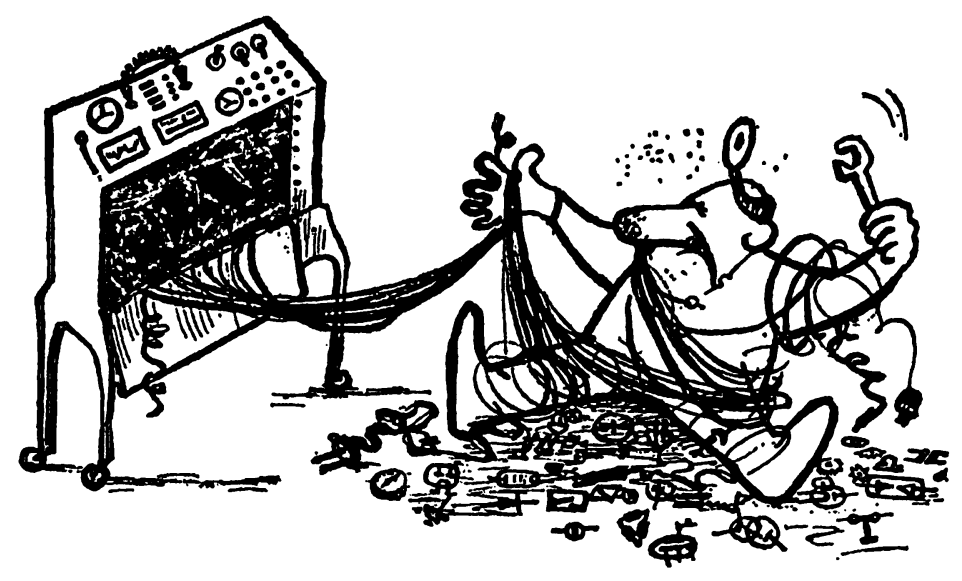

Die vielfachen ärztlichen Probleme einer Umbruchzeit in der Medizin finden in dieser Neuerscheinung bildhaften Ausdruck. Der zeichnerisch begabte Arzt schont seine Standesgenossen nicht, hilft ihnen indessen zu klarerer Einsicht in die Lage. Ein hervorragendes Geschenkwerk: von Arzt zu Arzt, vom dankbaren Patienten für den Behandler, von der Helferin oder Schwester für den Chef.

\section{F. LEHMANNS VERLAG MÜNCHEN}

\section{Dr. Armin Schäffer}

\section{Arzt aus Leidenschaft}

Heiteres und Kritisches

aus der großen weißen Welt

1972, 136 Seiten, Großformat, 75 Karikaturen Leinen DM 48,-

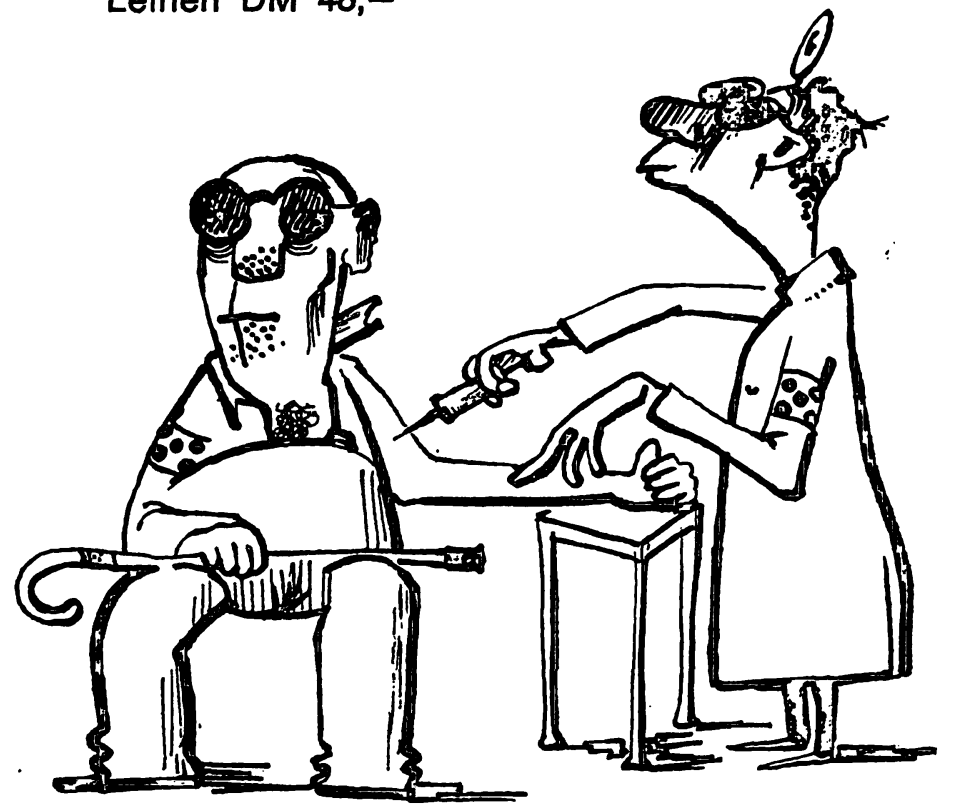

\section{BIOCHIMIE}

Edité par la Société de Chimie Biologique

tel est le titre

sous lequel paraîtra à partir de 1971

le „BULLETIN DE LA SOCIÉTÉ DE CHIMIE BIOLOGIQUE“

\section{SECRÉTARIAT'}

de la Société de Chimie Biologique

J. P. EBEL, Secrétaire Général (Relations Extérieures)

R. PERLES, Secrétaire Général

\section{REDACTION}

F. GROS, Secrétaire scientifique

F. PERCHERON, Secrétaire à la Publication

J. NUNEZ, Secrétaire à l'Information

Y. RAOUL, Secrétaire à l'Edition

SECRETARIAT et REDACTION: 4 Avenue de l'Observatoire, PARIS $6^{\circ}$ 12 FASCICULES

ABONNEMENTS: FRANCE et ZONE FRANC: 150 ffrcs - BELGIQUE: 1.687,- frcs - AUTRES PAYS: 186,- ffrcs

MASSON et Cie, Editeurs $\cdot 120$ Boulevard St Germain · PARIS Géme 


\section{W Walter de Gruyter Berlin.New York}

\section{Neuerscheinungen}

\section{Hilde Götz}

\section{Immunologische Plasmoprotein- Diagnostik}

Oktav. XII, 256 Seiten. Mit 106 Abbildungen. 1973. Gebunden DM 96, -

Das Buch vermittelt Methodik und klinischen Anwendungsbereich der wichtigsten immunologischen Untersuchungstechniken zur Differenzierung menschlicher Plasmaproteine. Es werden die Immunelektrophorese (Grabar und Williams bzw. Scheidegger) mit reichlichem Anschauungsmaterial und Interpretationshilfen, die zweidimensionale Doppel-Immundiffusion (Ouchterlony) sowie die einfache, radiale Immundiffusion im Agargel (Mancini und Mitarb.) dargelegt.

\section{Hilde Götz}

\section{Antigenität von Tumorproteinen}

Okłav. XII, 126 Seiten mit 19 Abbildungen und 6 Tabellen. 1972 Gebunden DM 60,-

Die Monographie versucht, aus der Komplexität der Probleme der Immunonkologie Wesen und klinische Bedeutung der Antigenität von Tumorproteinen darzulegen.

\section{W Walter de Gruyter Berlin.New York}

Neuerscheinung

\section{Fuchs - Freiwald}

\section{Allgemeine und anorganische Chemie}

Einführung in die Grundlagen für Mediziner, Naturwissenschạftler und Chemie-Nebenfächler

Von Prof. Dr. JOACHIM FUCHS und Dipl.-Chem. WILFRIED FREIWALD, Institut für Anorganische Chemie der Freien Universität Berlin

Groß-Okłav. VI, 174 Seiten. 1972. Kartoniert DM 12,80 ISBN 3110042436

Der ,Lerntext" soll in kürzester, aber dennoch verständlicher Form die wichtigsten Grundlagen der anorganischen und allgemeinen Chemie darstellen. Dabei ist der "Lernstoff" auf ein Minimum beschränkt, das Hauptgewicht wird auf ein Vermitteln allgemeiner Gesetzmäßigkeiten und Zusammenhänge gelegt.

Nach Kapiteln über Atombau und Periodensystem wird die Chemie der Elemente, geordnet nach den Gruppen des Periodensystems, besprochen, wobei an prägnanten Beispielen Gebiete aus der allgemeinen und physikalischen Chemie (Reaktionskinetik, Thermodynamik, Elektrochemie) in einfacher Form behandelt werden. Der Aufbau ist so gewählt; daß die Eigenschaften und Reaktionen der Stoffe mit Hilfe der jeweils bereits erklärten theoretischen Grundlagen anschaulich verständlich sind. Schon nach einem einmaligen intensiven Durcharbeiten des Stoffes wird ein hoher Lerneffekt erzielt.

Das Buch ist speziell für Studenten mit Chemie als Nebenfach (Mediziner, Biologen, Physiker u. a.) gedacht. Es sollte aber auch für Studenten der Chemie als Hauptfach und für Chemotechniker an Ingenieurakademien und Fachhochschulen in den Anfangssemestern' von Nutzen sein, umi zu erkennen, welche Gebiete der Chemie, die in ausführlichen und umfangreichen Lehrbüchern behandelt werden, von besonderer Wichtigkeit sind. 